\title{
Presynaptic Inhibition of Identified Wind-Sensitive Afferents in the Cercal System of the Locust
}

\author{
G. S. Boyan \\ Molecular Neurobiology Group, Research School of Biological Sciences, Australian National University, Canberra City, \\ A.C.T. 2601, Australia
}

The paired cerci located at the tip of the locust abdomen bear a large number of wind-sensitive filiform hairs, each of which sends an axon via the cercal nerve into the terminal ganglion of the CNS. The filiform afferents fire bursts of action potentials when their hairs are displaced by wind or mechanical stimuli. Filiform axon terminals in the CNS are depolarized concomitantly with the discharge of another type of unit (a primary afferent-depolarizing, or PAD, unit) recorded in the cercal nerve. The instantaneous spike frequency of PAD unit discharges matches the evoked depolarization very closely, and during such depolarizations spike amplitudes in the filiform afferent terminals are reduced by up to $55 \%$. Depolarizing current pulses injected into the axonal terminals of an identified filiform afferent evoke spikes that are blocked by the PAD unit, probably via an intercalated interneuron. The PAD unit makes a monosynaptic connection with only one of the 4 giant interneurons (GIN 2) on each side of the terminal ganglion, and indirect connections with 2 others. Depolarizing current pulses injected into the neuropilar segments of GINs evoke fewer spikes when the PAD unit is active, consistent with the PAD unit's mediation of conductance changes in postsynaptic cells. Iontophoretic injection of Lucifer yellow shows the PAD unit to be an afferent with axon terminals overlapping those of filiform afferents and posteriorly directed branches of interneurons such as GIN 2 in the CNS. Passive movements of a cercus, monitored with a position transducer, show that the PAD unit fires discrete bursts during cercal displacement. The PAD unit most probably has its soma and dendrites in tissue spanning the cercal base. By responding to cercal movements sufficient to also activate filiform hairs, and by mediating conductance changes in both the presynaptic terminals of filiform afferents and the postsynaptic membranes of interneurons, the PAD unit desensitizes a pathway to movement-generated afferent input, and ensures that the locust remains sensitive to external wind stimuli.

The efficiency with which a particular sensory system discriminates afferent information from environmental noise can contribute significantly to an animal's chances of survival. A major

\footnotetext{
Received March 17, 1987; revised Oct. 23, 1987; accepted Nov. 27, 1987.

I thank Drs. E. E. Ball and D. N. Reye for their comments on this manuscript, and Dr. P. Bräunig for drawing my attention to several important references.

Correspondence should be addressed to Dr. G. S. Boyan, Molecular Neurobiology Group, Research School of Biological Sciences, Australian National University, P.O. Box 475, Canberra City, A.C.T. 2601, Australia.

Copyright (C) 1988 Society for Neuroscience $0270-6474 / 88 / 082748-10 \$ 02.00 / 0$
}

source of noise in sensory information processing is the inadvertent activation of sensory receptors caused by the animal's own movement (von Holst and Mittelstaedt, 1950). Thus, synaptic mechanisms that distinguish afferent input produced by the animal's own movement from that originating in the environment, such as common mode rejection (Montgomery, 1984), corollary discharge (or efference copy) (Johnstone and Mark, 1971; Murphey and Palka, 1974; Delcomyn, 1977; Delcomyn and Daley, 1979; Bell, 1981), efferent inhibition (Russell and Roberts, 1974), lateral inhibition (O'Shea and Rowell, 1975; Fahrenbach, 1985), excitation-dependent inhibition(Palka, 1967, 1969), and presynaptic inhibition (Eccles et al., 1962; Levy, 1977; Ryall, 1978; Nicoll and Alger, 1979), are widely distributed throughout the animal kingdom, and have been intensively studied.

Among invertebrates, presynaptic inhibition has been shown to play a role in coordinating the activity of initiating neurons with proprioceptive input in the evasive jump of the locust (Pearson and Goodman, 1981), and in protecting central neurons of the escape circuitry in both the crayfish (Krasne and Bryan, 1973; Kennedy et al., 1974; Bryan and Krasne, 1977; Kirk and Wine, 1984; Kirk, 1985) and cricket (Levine and Murphey, 1980) from habituation. Interneurons producing presynaptic inhibition or primary afferent depolarization have now been identified in the cat (Solodkin et al., 1984) and in several invertebrates (locust: Pearson and Goodman, 1981; crayfish: Kirk and Wine, 1984; Glantz et al., 1985; Kirk, 1985; WangBennett and Glantz, 1985, 1986; Aplysia: Byrne, 1980; Kretz et al., $1986 \mathrm{a}, \mathrm{b})$.

The 2 dorsal antenna-like cerci of the locust (approximately $2 \mathrm{~mm}$ in length in the male) project posteriorly from the terminal segment of the abdomen (Fig. 1 $A$ ), and each cercus bears approximately 200 filiform hairs (Sihler, 1924; Thomas, 1965; Rozhkova et al., 1984), which are moved by air displacements such as wind and low-frequency sound. The axons of filiform hairs project into the terminal ganglion of the CNS, where they activate a number of postsynaptic cells, among them 4 giant interneurons (Cook, 1951; Seabrook, 1971; Boyan et al., 1986). In locust flight, oncoming wind and wind generated by the wings have both been shown to exert powerful phasic effects on the flight oscillator (Horsmann et al., 1983). Air currents are also generated during cricket stridulation (Kämper and Dambach, 1985) and cockroach walking (Plummer and Camhi, 1981), and filiform hairs on the cerci detect these air currents (Orida and Josephson, 1978; Plummer and Camhi, 1981; Kämper, 1984) and mediate phasic effects on the motor output (Dambach et al., 1983). The cerci have been shown to trigger kicking (Huber, 
1965), jump behavior (Hoyle, 1958), to initiate flight (Boyan et al., 1986), and to play a role in steering during flight (Fraser, 1977; Altman, 1983).

In this paper I demonstrate that, in the locust, presynaptic inhibition of identified filiform hair afferents occurs via primary afferent depolarization (PAD) when wind is directed at the cercus, or when the cercus is moved passively. The PAD is evoked by another rcceptor, a PAD unit, which is sensitive to movement of the cercus through space. I then describe the postsynaptic effects of the PAD unit on giant interneurons of the terminal ganglion and identify a giant interneuron that receives input directly from the joint receptor. During behavior such as free flight, the locust cercus moves as a result of both oncoming wind and the animal's own body movements. I propose that, by suppressing filiform afferent activity arising from cercal movement alone, presynaptic inhibition contributes to the locust's ability to discriminate activation of filiform hairs by an external stimulus from that arising from the animal's own movements.

\section{Materials and Methods}

Animals. All experiments were performed on cultured adult Locusta migratoria at lcast onc wcek postccdysis. Males werc preferred because of the ease of dissection of the terminal abdominal segment. In spite of the sexual dimorphism of the cerci, the units described here were present and responded similarly in both sexes. The results reported below were obtained from a total of 63 preparations.

Dissection. Animals were mounted dorsal side up on a styrofoam board after the wings and legs had been removed. A dorsal midline incision, the length of the abdomen, exposed the gut and reproductive organs, which were removed, and the ventrally located abdominal chain of ganglia. The cercal nerves (N10), which run in to the terminal ganglion, were freed of surrounding tissue from the ganglion up to the point at which they entered the cerci. To restrict synaptic input to the terminal ganglion to that originating from the cercal afferents under study, all branches from the cercal nerves within the abdominal cavity and all peripheral nerve roots of the terminal ganglion were severed. This dissection did not, however, involve branches of the cercal nerve at the base of the cercus, so that the motor innervation of the cercus remained intact. Care was taken not to disturb the extensive ventral tracheation of the terminal ganglion as the ganglion was lifted onto a silver platform for support during intracellular recording. The entire abdominal cavity was flooded with saline (in mM: $\mathrm{NaCl}, 147 ; \mathrm{KCl}, 10 ; \mathrm{CaCl}_{2}, 4 ; \mathrm{NaOH}$, 3; HEPES, 10), which was regularly drained and replaced. The animal continued to ventilate throughout the course of the experiment. All experiments were conducted at room temperature $\left(22-23^{\circ} \mathrm{C}\right)$.

Stimulation and extracellular recording. Wind stimuli were delivered to each cercus via a Pasteur pipette with an aperture small enough to ensure that only one cercus was stimulated at a time (this was confirmed by simultaneously recording from both afferent nerves during stimulation). Wind strengths were adjusted so as to cause the longitudinal filiform hairs to deflect. In some cases, individual filiform hairs could be stimulated in this way while recording from their terminals in the CNS (see Results), but in general the filiform hairs were stimulated mechanically by deflecting them singly with a fine glass rod (monitored under a dissecting microscope). Activation of the PAD unit was also achieved by mechanical stimulation of filiform hairs, either singly, with short-duration (10-100 msec) stimuli in some preparations, or as a group, mainly on the proximal dorsolateral part of the cercus. The 2-dimensional position of a cercus in space was monitored with a Sandeman (1968) position transducer. A high-frequency sinusoidal signal was applied to a fine $10 \mu \mathrm{m}$ silver wire glued to the tip of the cercus, which then acted as a wand moving between 2 plates connected to the differential inputs of an amplifier. The output of the transducer was linear over the range of cercal movements tested; the rise and fall times of the output were proportional to the velocity of cercal motion; and the trace deflection (upward or downward) indicated the direction of motion.

Bipolar $75 \mu \mathrm{m}$ silver wire hook electrodes were placed on each cercal nerve and on the ventral nerve cord anterior to the terminal ganglion, in order to monitor ongoing electrical activity. The electrodes on the cercal nerves established the sensitivity of the preparation to wind stimuli, provided a physiological monitor of the duration of the wind stim- uhus, and gave an extracellular correlate of intracellularly recorded potentials from afferent terminals in the CNS. Action potentials recorded via all the bipolar electrodes were differentially amplified and stored on magnetic tape along with the intracellular record.

The 3 sets of hook electrodes were also used to electrically stimulate, via a stimulus isolation unit, either afferent fibers in each of the cercal nerves or the axons of neurons ascending from and descending to the terminal ganglion in the ventral nerve cord. Appropriate voltages of stimulation produced a single volley arriving at the terminals of afferents or interneurons, as previously described (Pearson et al., 1985; Boyan, 1986).

Intracellular recording and staining. Intracellular recordings were made from the neuropilar segments of giant interneurons or from the terminal arborizations of cercal afferents in the terminal ganglion. All recordings were made with thin-walled glass micropipettes pulled on a BrownFlaming P-77 puller (Sutter Instruments, San Francisco, CA), which provided very low resistances of only $20-40 \mathrm{M} \Omega$ when the tips were filled with a $5 \%$ Lucifer yellow-CH solution dissolved in $0.1 \mathrm{M} \mathrm{LiCl}$. The electrodes were backfilled with a $0.5 \mathrm{M} \mathrm{LiCl}$ solution and introduced into the ganglion through an untreated neural sheath. Intracellular penetrations of interneurons were accompanied by a $40-60 \mathrm{mV}$ drop in potential and the onset of synaptic activity. Neural potentials were amplified by a DC amplifier with current-passing facility, displayed on an oscilloscope, and stored on magnetic tape. A silver wire inserted into the head capsule served as the indifferent electrode.

At the completion of every experiment, Lucifer yellow dye was iontophoretically injected into the recorded cell using constant 4-5 nA hyperpolarizing current for 5-10 min, while the membrane potential was continuously monitored. Immediately following dye injection, the terminal ganglion and attached cercal nerves were dissected from the preparation, fixed in a buffered ( $\mathrm{pH} 7.4) 4 \%$ formaldehyde solution for no longer than $30 \mathrm{~min}$, dehydrated, and cleared in methyl salicylate. Stained cells were photographed in whole-mount on a fluorescence microscope and then drawn using a camera lucida.

\section{Results}

\section{Normal activity in filiform hair afferents}

Each of the paired cerci (Fig. $1 A$ ) is covered by 2 main hair types - the long (up to $500 \mu \mathrm{m}$ ) and thin filiform hairs, of which there are up to 200 , mainly on the proximal parts of the cercus, and the shorter and stouter trichoid hairs (Fig. 1B). It is primarily the filiform hairs that respond to wind and activate the giant interneurons in the terminal ganglion. Intracellular recordings from the axonal arborizations of filiform hairs in the terminal ganglion of the CNS revealed high-frequency phasic/ tonic bursts of spikes in response to both wind and mechanical displacement of cercal hairs (Fig. $2 A$ ). The filiform hair being recorded from was identified each time by determining its location on the cercus, and by iontophoretic injection of Lucifer yellow into the terminal projections (Fig. 2B), although dye never progressed sufficiently far in a retrograde direction to also stain the cell body and dendrite on the cercus. The terminals of all filiform hairs recorded in this study showed a very similar morphology, namely, a dense mass of branches ventral and medial in the ganglion, with boutonlike densities on the finer branches, and an arborization that remained ipsilateral to the cercus of origin.

The cerci of the locust are motile and have an associated musculature (Uvarov, 1966). Movements of the animal under natural conditions could potentially create air currents sufficient to stimulate the filiform hairs on the cercus, as occurs during walking in cockroaches and crickets (Delcomyn, 1977; Orida and Josephson, 1978; Delcomyn and Daley, 1979). To test whether active movements of the abdomen and cercus created sufficient air displacement to stimulate the filiform afferents, the ventral nerve cord (VNC) was stimulated immediately anterior to the terminal ganglion in 4 preparations. The cerci were monitored optically and the stimulus voltage adjusted so that a burst 


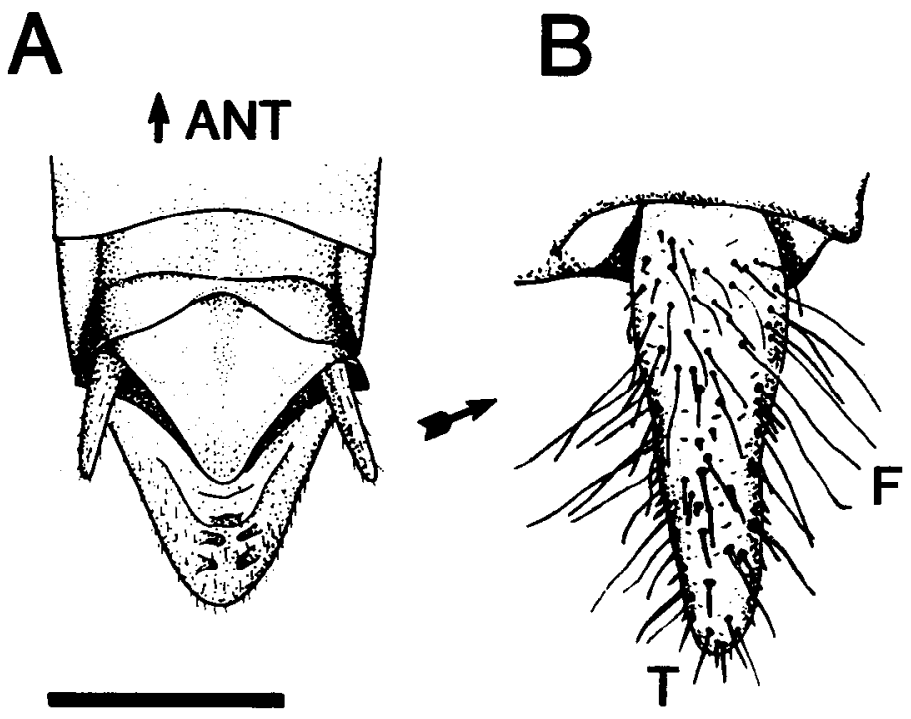

Figure 1. The posterior segments and cerci of an adult male locust. $A$, Drawing of the posterior segments of a male locust, viewed dorsally, and showing the 2 cerci projecting posteriorly and slightly laterally from the body. $B$, Drawing of a cercus from a male locust showing in dorsolateral view the long filiform hairs $(F)$ and shorter trichoid sensilla $(T)$. Scale bar, $2.5 \mathrm{~mm}(A), 0.7 \mathrm{~mm}(B)$.

of stimulation $(200 \mathrm{~Hz})$ just caused the abdomen and cerci to move. Two sets of recording electrodes were placed on one cercal nerve to monitor neural activity evoked as a result of stimulation. A puff of wind directed at the cercus being recorded from elicited a summed response from a number of filiform hairs and established the sensitivity of the afferent pathway (Fig. 3, arrow). The electrical stimuli that followed elicited large-amplitude spikes from several units, and the form of the responses was almost identical to that of filiform hairs initially responding to wind (Fig. 3). The 2 sets of recording electrodes showed that some of the spikes evoked by cercal movement had the same conduction velocity as did filiform afferents stimulated by wind $(1.4 \mathrm{~m} / \mathrm{sec})$. Active movement of the abdomen and cerci therefore created sufficient wind displacement to stimulate filiform hair afferents,

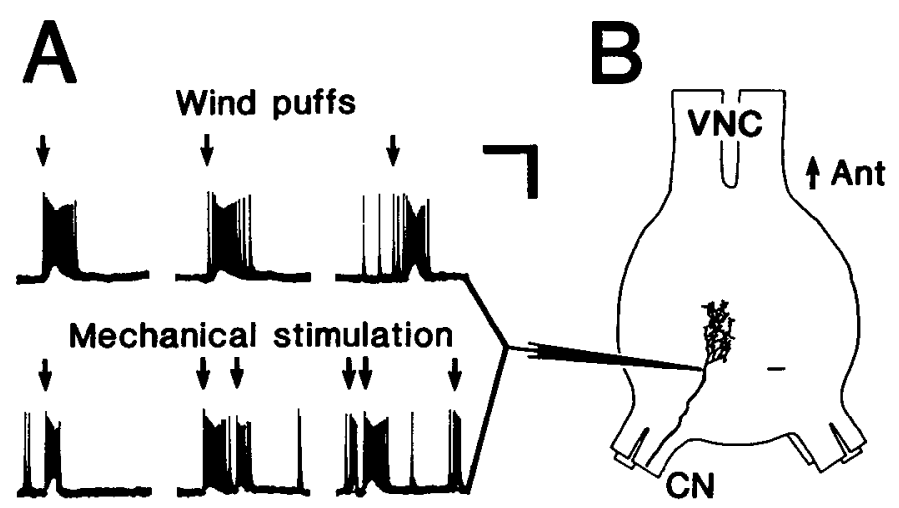

Figure 2. Spikes recorded intracellularly from axon terminals of a filiform afferent in the CNS. $A$. Stimulation of an individual filiform hair by wind and mechanical displacement (onset indicated by arrows) evoke similar phasic/tonic bursts of spikes. $B$, Drawing from wholemount of the terminal arborizations of the filiform afferent recorded in $A$ after injection with Lucifer yellow. The afferent was one of the group marked $F$ on the cercus in Figure $1 B . C N$, cercal nerve; $V N C$, ventral nerve cord. Scale bars, $A$ (vertical), $20 \mathrm{mV}$, (horizontal) $100 \mathrm{msec} ; B$, $45 \mu \mathrm{m}$.

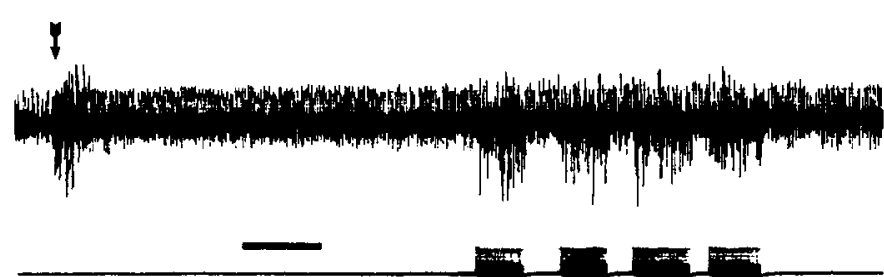

Figure 3. Movement of cerci evoked by electrical stimulation of the VNC is sufficient to activate filiform afferent hairs. A wind puff directed at a cercus evokes a summed response from a number of filiform afferents recorded extracellularly in the cercal nerve (arrow, onset of excitation due to wind). Electrical stimulation ( $200 \mathrm{~Hz}$ bursts) of the VNC (lower trace) causes the cerci to move and evokes responses from filiform afferents that are very similar to those produced by the initial wind puff. Scale bar, $300 \mathrm{msec}$.

and the problem the locust faces is to distinguish between activation of filiform hairs due to an external stimulus and that due to cercal movement alone.

Wind puffs directed at a cercus always stimulated several filiform hairs and elicited a massed excitatory response in the afferent nerve of similar duration to that of the stimulus (Figs. $3,4 A$ ). In most preparations, wind puffs and the mechanical deflection of one or more filiform afferent hairs proximally on the cercus also released a prominent afterdischarge from a single unit (Fig. 4A). Such afterdischarges could vary, in the same preparation, from short bursts of spikes to long sequences that sometimes spanned the interval between successive stimuli (Fig. $4 A$ ). Short bursts of spikes from the unit also occurred spontaneously in the absence of any wind stimuli directed at the cercus. While the same unit could discharge in both short and long bursts in the one preparation, the longer bursts were selected for analysis here because their effects on the membrane potential of filiform afferents were more obvious. Regardless of their length, these spike sequences were only slowly adapting, as evidenced by the plot of instantaneous spike frequency (Fig. $4 B$ ). The unit producing the maintained discharge did not respond similarly to any of the filiform afferent hairs recorded from, which, in the same preparation, usually had larger spike amplitudes (recorded extracellularly), a very phasic discharge pattern, and higher stimulus threshold to cercal movement.

\section{Depolarization of filiform afferents (PAD)}

Intracellular recordings from the terminals of filiform afferents in the CNS consistently showed that a depolarization of their membrane accompanied the maintained discharge in the background cell (Fig. 5A). All filiform afferents tested were effective in releasing the maintained discharge of the background cell and thereby, in turn, in depolarizing a given filiform afferent. Activity in the filiform afferent being recorded was not a prerequisite for observing a depolarization of that cell, which showed that self-stimulation was not involved. It is important that only a stimulus that released the maintained discharge in the background cell also evoked depolarization of the filiform afferent membrane; such depolarizations were never recorded in the absence of activity in the background unit. The unit responsible for the maintained discharge in cercal nerve recordings is therefore almost certainly a primary afferent depolarization (PAD)producing unit, and will be referred to as such below. Finally, the depolarization of the afferent terminal lasted considerably longer than did the mechanical or wind stimulus evoking the maintained discharge in the PAD unit, but was consistently 
A

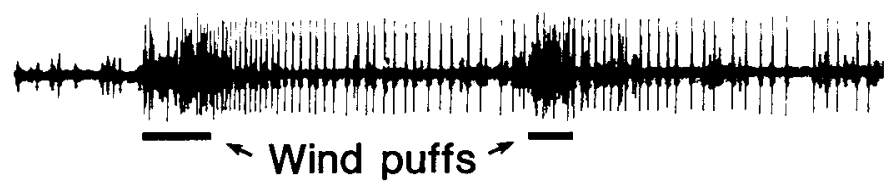

B

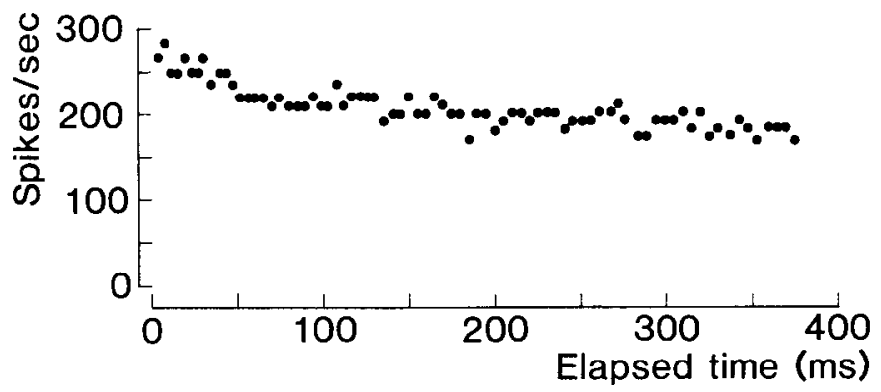

Figure 4. Extracellular recording of activity from a cercal nerve showing responses to wind puffs directed at a cercus. $A$, Wind puffs evoke a phasic burst of activity from many filiform afferents and a discharge from the PAD unit that is maintained after the stimulus ends, and can span the interval between stimuli. $B$, Plot of instantaneous spike frequency in a representative sequence of the PAD unit that was evoked by mechanical stimulation of filiform hairs on the cercus. Duration of first wind stimulus in $A, 70 \mathrm{msec}$.

shorter than the duration of the maintained discharge itself(Figs. 5, 6A).

Comparison of the instantaneous spike frequency of the PAD unit with the concomitantly evoked depolarization of the filiform afferent membrane showed that their time courses followed one another very closely (Fig. $5 B$ ). The depolarization rose rapidly to a peak of about $2 \mathrm{mV}$ over the first $30-50 \mathrm{msec}$, when spike rates were at their highest $(300-450 \mathrm{spikes} / \mathrm{sec})$, and then declined with the spike rate to a plateau level of $1.0-1.5 \mathrm{mV}$, which was not, however, maintained for the full duration of the afterdischarges recorded. This difference between the durations of depolarization of the filiform afferent and the spike discharge in the PAD unit suggests a polysynaptic pathway between them.

For the depolarization of filiform afferents to be considered physiological, it should cause a reduction in the amplitude of spikes in the terminals, and this was clearly the case (Fig. 6A). A comparison of the degree of depolarization with the resulting reduction in spike amplitudes showed that, as the depolarization increased, the amplitude of spikes could be reduced by up to $55 \%$, although the relationship was not linear (Fig. $6 B$ ). The $1.0-2.0 \mathrm{mV}$ depolarization evoked in the afferent terminal by the maintained discharge of the PAD unit described in Figure $5 B$ reduced spike amplitudes by $25-45 \%$ (not shown).

Electrical stimulation of the cercal nerve was used to activate both filiform afferents and the PAD unit. Stimulation evoked an initial spike in the filiform afferent terminal, and 5-7 msec later a depolarizing afterpotential $1.5-2.0 \mathrm{mV}$ in amplitude and 40-70 msec in duration (Fig. $7 A$ ). Spontaneous spikes occurring during this depolarizing afterpotential were also reduced in amplitude. Although the filiform afferents project into the terminal ganglion ipsilateral to their cercus (Fig. 2), interaction might still have occurred between filiform afferents, or PAD units, from each side. Electrical stimulation of ipsilateral and contralateral
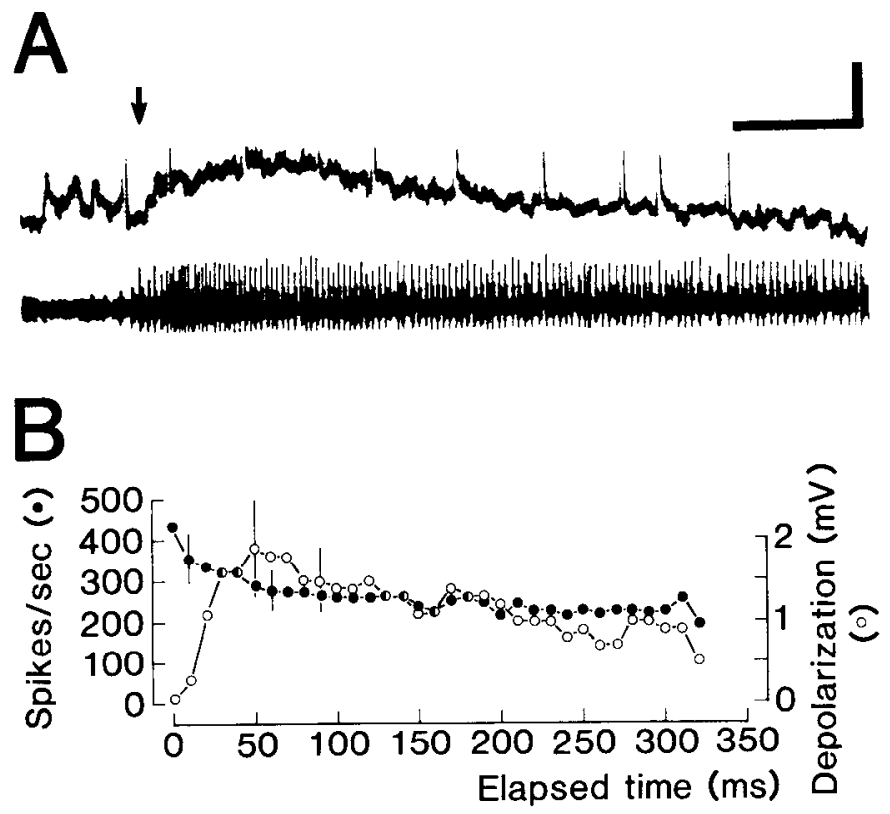

Figure 5. Primary afferent depolarization in a filiform afferent. $A$, Intracellular recording (upper trace) from the axon terminals of a filiform afferent showing depolarization evoked by the PAD unit, monitored extracellularly in the cercal nerve (lower trace). Mechanical stimulation of filiform hairs activated the PAD unit, as indicated by the arrow. Spikes have been cul of by the gain setting used to display the depolarization. $B$, Plot of instantaneous frequency of spikes in the PAD unit $(\bullet)$, and depolarization evoked simultaneously in the filiform afferent terminal (O). Points are the means of 4 sequences recorded in the same preparation, and representative error bars show 1 SD from the mean. Mechanical stimulation of filiform hairs elicited PAD unit activity, and sequences of approximately equal length were grouped for analysis. Similar results were obtained in 2 other preparations. Scale bars, $2 \mathrm{mV}$ (vertical), $100 \mathrm{msec}$ (horizontal).

cercal nerves in the same preparation established that only ipsilateral stimulation evoked both the initial spike in the filiform afferent and the depolarizing afterpotential attributable to the PAD unit (Fig. 7B).

\section{Identification of the PAD unit}

The cercal nerve is a mixed nerve containing motor as well as sensory units (Seabrook, 1970). To establish that the PAD unit is an afferent, the cercal nerve was severed as it entered the terminal ganglion. The PAD unit could still be activated by stimulation of filiform afferent hairs, which also showed that efferent activity did not evoke the response or contribute to its patterning (see also Fig. 12). The PAD unit of an intact preparation was then impaled in the terminal ganglion with a $\mathrm{mi}$ croelectrode, and a 1:1 correlation between the spike recorded in the CNS and that monitored extracellularly in the cercal nerve showed that the action potential propagated towards the CNS at $1.1 \mathrm{~m} / \mathrm{sec}(1.4$ and $1.3 \mathrm{~m} / \mathrm{sec}$ in 2 other preparations) (Fig. $8 A$ ). These data predict a latency of about $2-3 \mathrm{msec}$ for an action potential to reach the recording site in the CNS from the cercus. Iontophoretic injection of the Lucifer yellow dye via the microelectrode established that the PAD unit was an afferent entering the CNS via the cercal nerve and terminating in the same region as the axons of filiform hairs (Fig. 8B; cf. Fig. 2).

Several experiments aimed at determining the location of the cell body and dendrite of the PAD unit(s) were performed, but proved equivocal. Sectioning of nerves innervating the cercus 

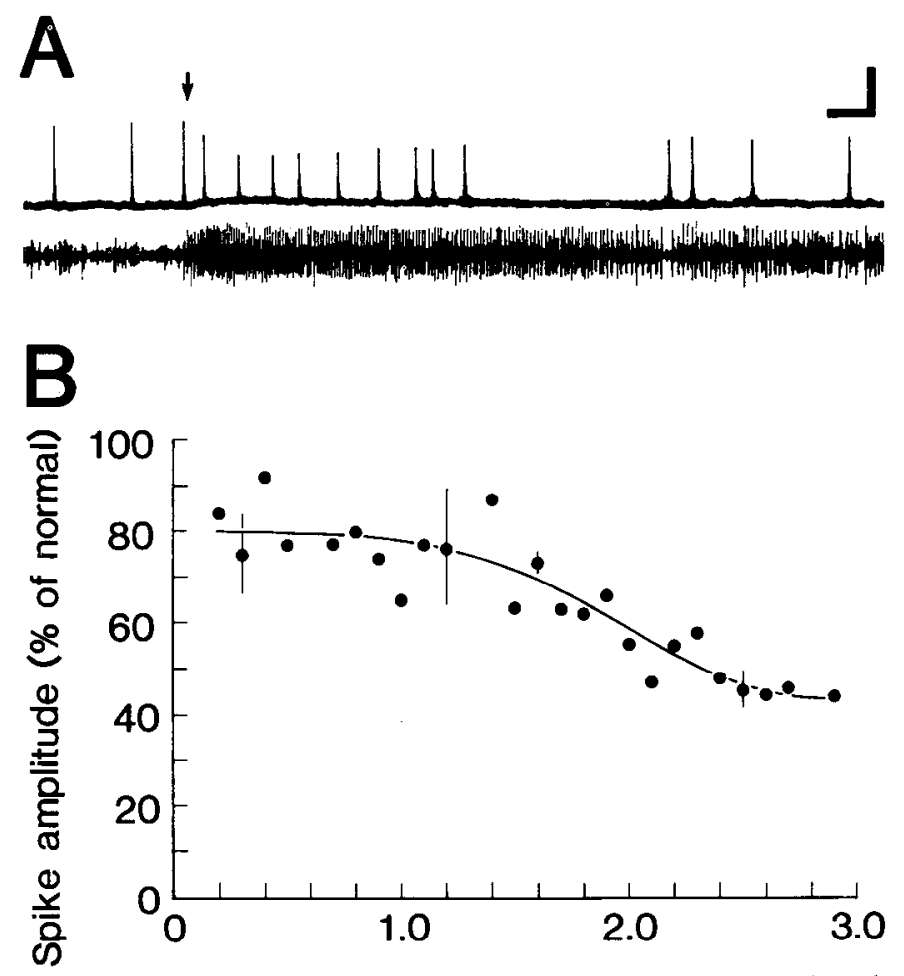

Membrane depolarization $(\mathrm{mV})$

Figure 6. Reduction in amplitude of spikes accompanies depolarization of filiform afferent terminals. $A$, Mechanical stimulation of filiform hairs (other than the one being recorded) activates the PAD unit (lower trace, onset indicated by arrow), which depolarizes the terminal membrane and reduces spike amplitudes in the intracellularly recorded filiform afferent (upper trace). Amplitudes of filiform afferent spikes recover as the spike rate in the PAD unit declines. $B$, Plot showing amplitudes of spikes for various levels of depolarization of the filiform afferent terminal evoked by the PAD unit. Points represent means of data from 7 sequences in one preparation and are expressed as a percentage of the mean spike height recorded in the absence of PAD unit activity. The same pattern was obtained in 2 other preparations. Scale bars, $20 \mathrm{mV}$ (vertical), $100 \mathrm{msec}$ (horizontal).

established that the PAD unit axon was located in the main nerve trunk entering the cercus itself, rather than in side branches innervating surrounding musculature, for example. Cobalt and Lucifer yellow backfills revealed previously undescribed groups of cell bodies and associated dendrites leading off the main nerve trunk in several regions around the cercal base, with all in a position to possibly monitor cercal movement. These included a strand receptor at the cercal base, as well as hairs at the cercal base that would be deflected by cercal movement. Since none of these cell groups could be unequivocally linked to the terminal projections stained in the CNS (Fig. 8), it is speculative to conclude that one particular group contributed to the response recorded centrally.

\section{Presynaptic inhibition of spikes}

To test whether spikes could also be presynaptically inhibited in the filiform afferent terminals, the membrane response of an identified filiform afferent was recorded as depolarizing current pulses were injected into its terminals and the PAD unit activated. Depolarizing current pulses were found to regularly elicit spikes in the terminal region (Fig. 9A). When the PAD unit was active, there was a clear reduction in the number of spikes elicited by the same current pulse (Fig. 9, $A, B$ ), consistent with
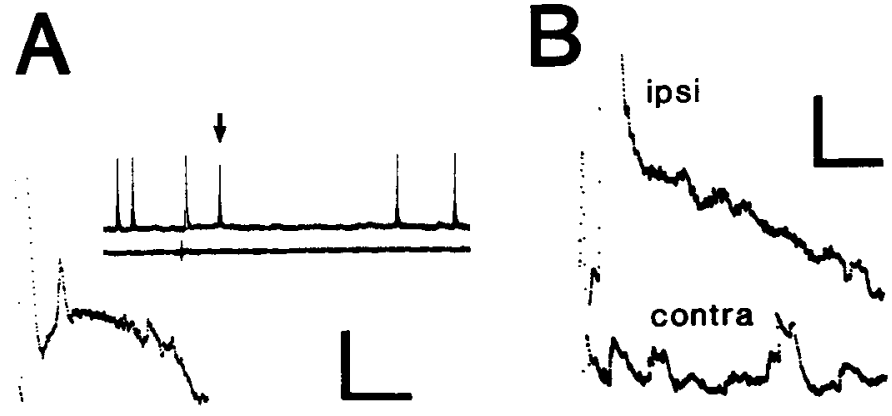

Figure 7. Depolarizing afterpotentials evoked in terminals of filiform afferents on electrical stimulation of the afferent nerve. $A$, Averaged record from 32 events shows an initial spike (full amplitude not shown) followed by a depolarization. Small peak on the depolarizing afterpotential results from averaging spontaneously occurring spikes. Inset, Oscilloscope trace with longer time course shows ongoing intracellular recording from a filiform afferent terminal (upper trace). Electrical stimulation of the afferent nerve (lower trace) evokes a spike and depolarizing afterpotential in the filiform afferent. A spontaneous spike occurs during the depolarizing afterpotential and its amplitude is reduced (arrow). $B$, Responses recorded from a filiform afferent terminal on electrical stimulation of the afferent nerve ipsilateral and contralateral to the location of the innervated hair on the cercus. Only ipsilateral stimulation evokes a spike and the depolarizing afterpotential. Data are from a different preparation from $A$, and traces are AC-coupled. Scale bars, $A, 1 \mathrm{mV}$ (vertical), $30 \mathrm{mV}$ (inset); $20 \mathrm{msec}$ (horizontal), $70 \mathrm{msec}$ (inset); $B, 1 \mathrm{mV}$, (vertical), $10 \mathrm{msec}$ (horizontal).

an increase in membrane conductance in the terminal region. When $\mathrm{Cl}^{-}$was injected for $5 \mathrm{~min}$ via the recording electrode into the terminals of a filiform afferent (not shown), this resulted in a depolarizing afterpotential of similar size and duration to that evoked upon electrical stimulation of the cercal nerve above.

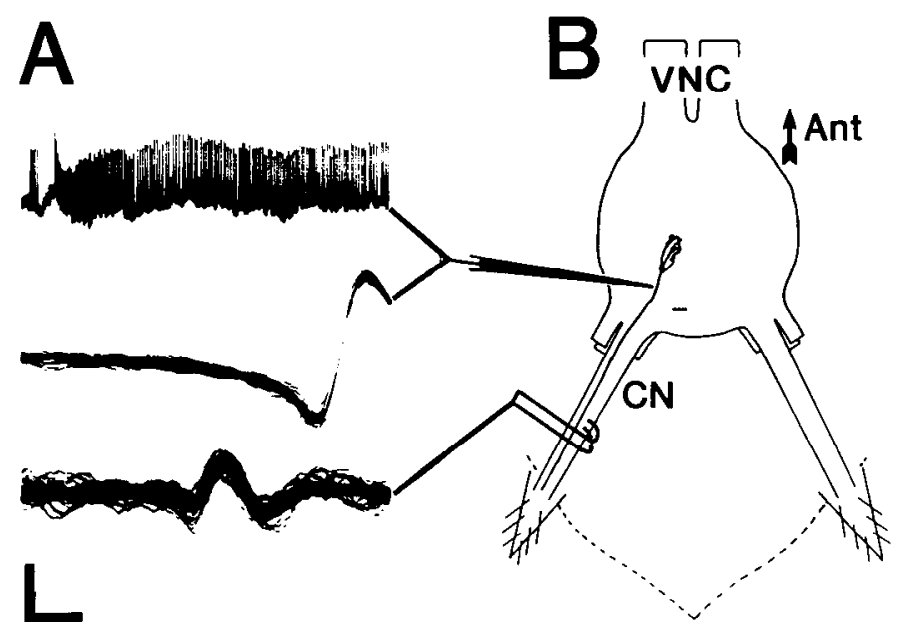

Figure 8. Identification of the terminal projections of the PAD unit in the CNS. $A$, Microelectrode recording of a PAD unit from its terminals in the CNS. Discharge in top trace was evoked by mechanical stimulation of filiform hairs on the cercus. Triggering from the PAD spike recorded by the microelectrode (middle trace) allowed the simultaneously recorded extracellular PAD spike to be identified in the cercal nerve (bottom trace) and the conduction velocity to be determined (1.1 $\mathrm{m} / \mathrm{sec}$ ). The tape recorder was replayed backwards and the trace reversed to obtain the record shown. All traces are displayed AC-coupled. $B$, Axonal projections in the terminal ganglion of the PAD unit recorded in $A$ stained with Lucifer yellow and drawn from whole-mount. Dashed line, schematic outline of the posterior tip of the abdomen. Scale bars, $A, 20 \mathrm{mV}$ (vertical, top trace), $8 \mathrm{mV}$ (middle trace), $200 \mathrm{msec}$ (horizontal, top trace), $0.8 \mathrm{msec}$ (middle and bottom traces); $B, 45 \mu \mathrm{m}$. 
A
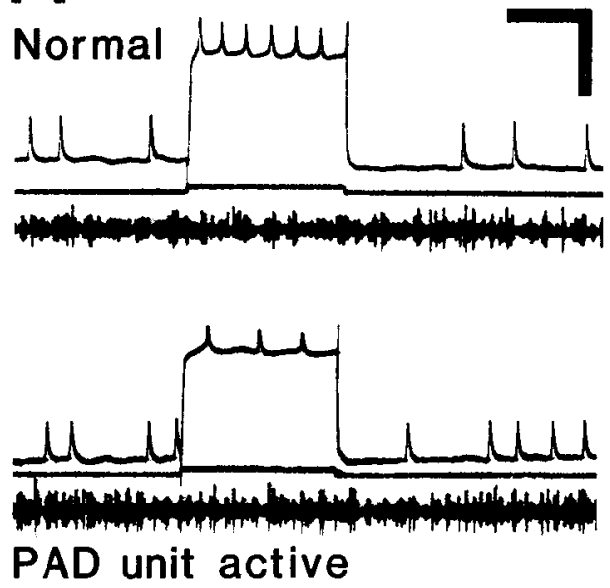

B

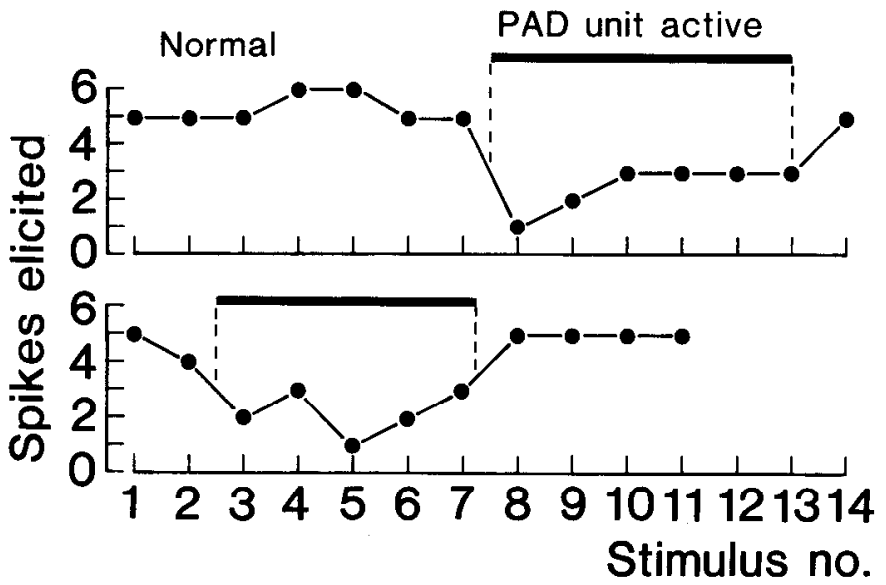

Figure 9. Presynaptic inhibition of spikes in a filiform afferent. $A$, Depolarizing current pulses $(3.5 \mathrm{nA})$ injected intracellularly into the terminals of a filiform afferent elicit spikes in the absence of PAD unit activity. Current pulses of the same amplitude elicit very few spikes when injected during discharge of the PAD unit in the same preparation. Amplifier was not properly balanced during current injection. $B$, Graphs showing number of spikes elicited in filiform afferent terminals by depolarizing current pulses $(3.5 \mathrm{nA})$, with and without concomitant PAD unit activity. Pulses of $100 \mathrm{msec}$ duration were presented at $1 / \mathrm{sec}$. Data are from 2 sequences in the same preparation. Scale bars, $40 \mathrm{mV}$ (vertical), $50 \mathrm{msec}$ (horizontal).

The depolarizing afterpotential remained for some minutes after cessation of current injection, and such an increased $\mathrm{Cl}^{-}$conductance could mediate the effects on spike amplitude and number in the filiform afferent terminals described above.

\section{Postsynaptic effects of the PAD unit}

Giant interneurons (GINs) have been associated with powerful behavioral sequences, such as escape, in many invertebrates (Dorsett, 1980). The locust has just 4 GINs in its wind-sensitive cercal system (Cook, 1951; Seabrook, 1971), greatly simplifying any investigation of synaptic interactions.

Connections with postsynaptic cells. Of the 4 giant neurons in the locust terminal ganglion, the PAD unit made constant, shortlatency (probably monosynaptic) excitatory connections only with GIN 2. Activation of the PAD unit produced a considerable increase in excitatory input to GIN 2, and triggering the oscilloscope trace off of PAD spikes in each cercal nerve revealed that short-latency $(2.7 \mathrm{msec})$ EPSPs were evoked in the GIN by PAD unit activity in both cercal nerves (Fig. 10 $A$ ). The recorded GIN 2 was then injected with Lucifer yellow to reveal its morphology in the terminal ganglion. The recording site on this occasion was in the crossing segment, near its branch point with the posteriorly directed arborization on the soma side of the ganglion. At this recording site, the EPSP evoked by the ipsilateral (to the soma) PAD unit showed a steeper rise time, quicker decay time, identical latency, but smaller amplitude, than the

$\begin{aligned} & \text { Table 1. Mean amplitude and reduction in amplitude of EPSPs } \\ & \text { evoked in an interneuron by filiform afferents in } 2 \text { preparations }\end{aligned}$
$\begin{array}{llll}\text { Control } & \text { PAD unit active } & \begin{array}{l}\text { Reduction } \\ \text { in } \\ \text { amplitude } \\ \text { EP) }\end{array} \\ \begin{array}{l}\text { Prepara- } \\ \text { tion }\end{array} & \begin{array}{l}\text { EPS amplitude } \\ (\mathrm{mV})\end{array} & \begin{array}{l}\text { EPSP amplitude } \\ (\mathrm{mV})\end{array} & \begin{array}{l}\text { (\%) } \\ \hline 1\end{array} \\ 2 & 6.3 \pm 1.8(54) & 4.9 \pm 1.5(31) & 23 \\ 2 & 3.7 \pm 1.0(60) & 3.2 \pm 0.7(17) & 15\end{array}$

Figures in parentheses are the number of EPSPs sampled to obtain each mean. Errors represent $1 \mathrm{SD}$ of the mean. contralaterally evoked EPSP. GIN 2 and GIN 4 were the only giant cells tested in which discrete PSPs were recorded on activation of the PAD unit, and only in GIN 2 was there a 1:1 correlation between these PSPs and spikes from the PAD unit.

Change in amplitude of EPSPS. A predicted consequence of the presynaptic reduction in both spike amplitude and spike number in filiform afferent terminals (Figs. 5-7) was that transmitter release from the terminals should have been depressed when the PAD unit was active. This should, in turn, have been
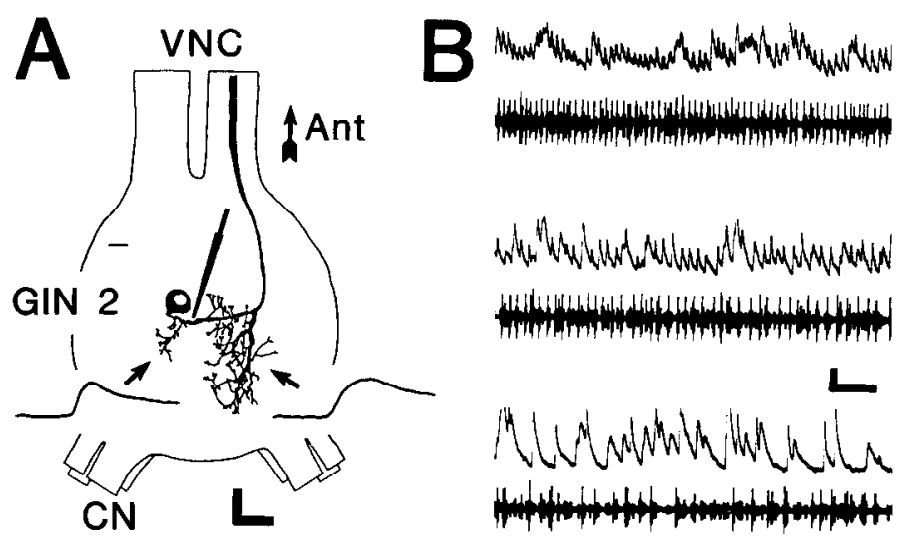

Figure 10. The PAD unit makes a constant, short-latency (probably monosynaptic) excitatory connection with GIN 2 in the terminal ganglion. $A$, The GIN 2, stained with Lucifer yellow and drawn from wholemount. Insets show the averaged EPSPs evoked in GIN 2 by the PAD unit on each side when the recording was made from the site indicated by the long arrowhead. Traces were triggered by PAD unit spikes. Short arrows indicate the probable branches of GIN 2 where the EPSPs on each side were evoked. $B$, Three segments from one sequence of PAD unit activity (lower traces) showing EPSPs evoked 1:1 in GIN 2. Note the progressive increase in both amplitude and frequency of EPSPs evoked by inputs other than the PAD unit, and the return of spontaneous spiking activity (full spike amplitudes not shown) as the spike rate in the PAD unit declines. Scale bars, $A, 2 \mathrm{mV}$ (vertical), $1 \mathrm{msec}$ (horizontal), $45 \mu \mathrm{m}$ (anatomical scale); $B, 4 \mathrm{mV}$ (vertical), $50 \mathrm{msec}$ (horizontal). 
Figure 11. Conductance changes mediated by the PAD unit in a representative postsynaptic neuron. Depolarizing current pulses $(2 \mathrm{nA})$ injected into GIN 4 (upper trace) elicit fewer spikes during concomitant PAD unit activity (seen in bottom trace, onset indicated by arrow) than in its absence. Bottom trace is from the cercal nerve. Scale bars, $20 \mathrm{mV}$ (vertical; intracellular recordings only), $100 \mathrm{msec}$ (horizontal).

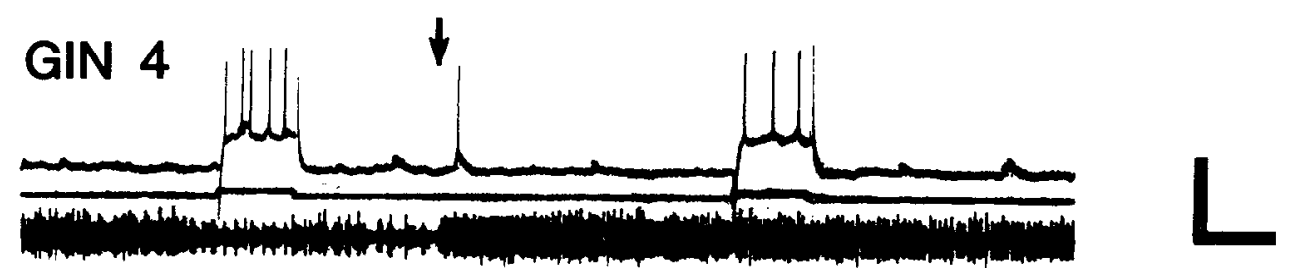

reflected in a reduction in the amplitudes of EPSPs evoked in postsynaptic cells by other sources of input, such as filiform afferents. Some indication of this appears in recordings made from GIN 2 during a scquence of PAD unit activity (Fig. 10B). Such recordings show small-amplitude EPSPs correlated 1:1 with spikes of the PAD unit, and larger-amplitude EPSPs evoked by unidentified inputs, such as filiform afferents or other interneurons. As the spike frequency of the PAD unit progressively declined and evoked proportionately fewer EPSPs, both the amplitude and frequency of EPSPs attributable to inputs other than the PAD unit increased. Spontaneous spiking activity also resumed. Recordings made from another interneuron show, when quantified (Table 1), that the EPSPs normally evoked by filiform afferents were reduced in amplitude (by 23 and $15 \%$ in 2 preparations, respectively) when the PAD unit was active.

Conductance changes. The PAD unit could also be shown to mediate changes in the membrane conductance of GINs. When
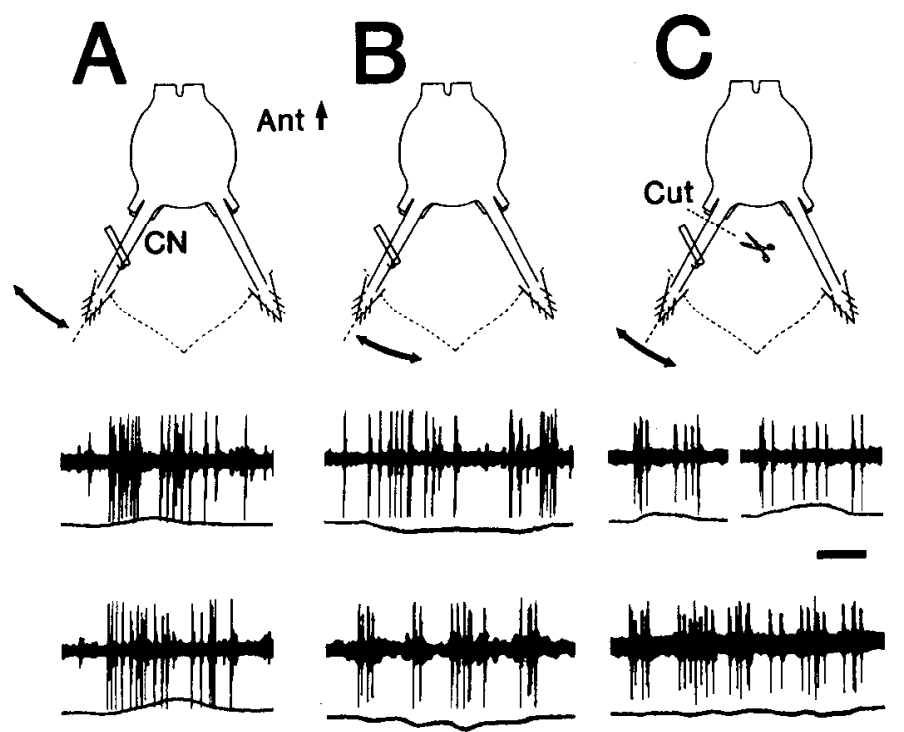

Figure 12. The PAD unit monitors cercal movement. A, Passive movement of the cercus away from the body midline evokes activity in the PAD unit recorded extracellularly in the cercal nerve $(C N)$. Cerci were displaced by up to $15^{\circ}$ from their resting position. Spikes occur primarily on the rising and falling phases of the motion recorded by the position transducer (lower traces), rather than when the cercus is stationary. A steeper ramp evokes a higher spike rate. $B$, With the cercus now moved towards the body midline (transducer output trace is inverted), spikes clearly occur on the ramps rather than during maintained displacement. Stepped movements evoke discrete groups of spikes. $C$, Severing the cercal nerve as it enters the CNS does not affect the patterning of the response seen in $A$ and $B$. Rise and fall times of the transducer output measure velocity of motion, and amplitude is linear with the extent of cercal displacement. These results were repeatable in 4 preparations. Scale bar, $100 \mathrm{msec}$. depolarizing current pulses were injected into GIN 4 (Fig. 11), the number of spikes elicited by a given current pulse was reduced from a normal average of 4-5 spikes to 2-3 spikes/pulse for the duration of concomitant PAD unit activity. These cffects were also seen in other GINs of the cercal system.

\section{$P A D$ unit responds to movement of the cercus}

The spiking response of the PAD unit consisted of slowly adapting spike trains of varying duration. The discharges were more often sustained when elicited by mechanical stimulation of filiform hairs on the cerci (Figs. 4, 5), and might reflect reflex movements-perhaps of the abdomen as well as the cercusover a longer time course. However, wind puffs to the cerci often elicited very discrete bursts of activity in the PAD unit, which then suggested the activity of a receptor monitoring rapid deflections of the cercus by wind. This was tested by moving a cercus passively through various angles towards and away from the body midline while recording the PAD unit extracellularly in the cercal nerve, and simultaneously monitoring cercal position with a position transducer. With the cercus at rest, there was no firing of the PAD unit to indicate that a load was being placed on the cercus by the transducer. Passive movements of the cercus away from (Fig. 12A) and towards (Fig. 12B) the midline resulted in a discharge of the PAD unit, with a clear grouping of spikes. Spikes occurred on the rising and falling phases of the position trace, when the cercus was moving, rather than at the peak, when the cercus was momentarily stationary (Fig. 12A), or when the cercus was maintained in a particular position (Fig. 12B). Spike frequencies were proportional to the steepness of ramp displacements (Fig. 12A). Stepped displacements of the cercus therefore elicited discrete groups of spikes (Fig. 12B). These patterns of PAD unit activity did not change when the cercal nerve was severed at the ganglion, precluding any contribution from efferent activity (Fig. 12C). Such passive movements of the cercus, on the other hand, rarely evoked the maintained type of discharge of the PAD unit that resulted from mechanical stimulation of filiform afferent hairs.

\section{Discussion}

The axon terminals of identificd wind-scnsitive filiform hairs in the CNS of the locust were depolarized when a PAD unit sensitive to displacement of the cercus discharged (Figs. 5, 12). The depolarization reduced spike amplitudes in the afferent terminals by up to $55 \%$ (Fig. 6), and activation of the PAD unit also presynaptically blocked spikes initiated in the filiform afferent terminals by current injection (Fig. 9). The resulting depression of transmitter release probably accounted for the 15$23 \%$ reduction in amplitude of evoked EPSPs in a postsynaptic cell (Table 1). Activation of the PAD unit was sufficient for both the depolarization of filiform afferent terminals (Fig. 5) and the 
decrease in spike numbers elicited on current injection into a postsynaptic neuron (Fig. 11).

The projections of the PAD unit in the CNS are ipsilateral to the cercus of origin (Fig. 8), and terminate in the glomerulus formed by the terminals of filiform afferents (Fig. 2). It is not clear, however, whether the presynaptic effects described above reflect a direct interaction between receptors or are mediated by a PAD-producing interneuron. Three factors suggest that an intercalated interneuron was involved: (1) The latency of 5-7 msec from electrical stimulation of the cercal nerve to depolarization of the filiform afferent was considerably longer than the latency predicted on the basis of conduction velocity (2-3 msec) and synaptic delay alone (Fig. 7). (2) Both the amplitude and duration of the depolarizing afterpotential evoked in filiform afferents upon a single electrical stimulation of the afferent nerve (Fig. 7) were greater than might be expected if the stimulus activated only the PAD unit once. And (3) discrete PSPs were absent from recordings of filiform afferent terminals when the PAD unit was active (Fig. 5), although the input synapses may have been distant from the recording site. Input as well as output synapses have been reported on the primary afferent terminals of other wind-sensitive hairs in the locust (Watson and Pflüger, 1984), providing an anatomical basis for the type of direct excitatory and inhibitory interactions between mechanosensory afferents described by Eckert (1961), Baylor and Nicholls (1969), Coro and Pérez (1983), and Blagburn and Sattelle (1987).

The terminals of the PAD unit in the CNS also overlap the posteriorly directed branches of several interneurons, including those on the soma side of GIN 2, where it is likely that some of the input synapses are located (Fig. 10A). Evidence supporting a monosynaptic input to GIN 2 from the PAD unit includes the fact that the EPSPs were evoked 1:1 by PAD unit spikes in the cercal nerve, and that the latency of evoked EPSPs $(2.7 \mathrm{msec})$ closely matched the latency of PAD spikes in the CNS predicted on the basis of conduction velocity $(2-3 \mathrm{msec})$.

The mechanism of spike generation in filiform afferent terminals and its involvement in presynaptic inhibition were not investigated in this study. The PAD unit depolarized the presynaptic membrane, as well as reducing spike numbers elicited by current injection at both pre- and postsynaptic sites. These effects are consistent with an increase in pre- and postsynaptic membrane conductance, perhaps mediated by $\mathrm{a} \mathrm{Cl}^{-}$current such as the one activated upon injection of $\mathrm{Cl}^{-}$into the filiform afferent terminals (see Results). If so, this would imply that a depolarizing IPSP was responsible, at the least, for the effects on spike amplitude at the presynaptic terminal. Presynaptic inhibition in the cercal system of the cockroach has been shown to be mediated by an increased $\mathrm{Cl}^{-}$conductance (Callec, 1985), as well as by delayed rectification mediated by voltage-gated $\mathrm{K}^{+}$ channels (Blagburn and Sattelle, 1987). The experiments reported in the present study cannot distinguish between these 2 possible mechanisms.

\section{Functional attributes of the PAD unit}

The cerci have been shown to play critical roles in the behaviors of several insects. Wind stimulation of filiform afferent hairs on the cerci can elicit jumping (Hoyle, 1958) and flight (Boyan et al., 1986) in the locust, kicking in the cricket (Huber, 1965), escape running in the cockroach (Camhi, 1980); the cerci are vital for flight stabilization in both the cockroach (Fraser, 1977) and locust (Altman, 1983). Cercal position, in particular, has been shown to determine the directionality of GINs in some species (Rozhkova, 1980; Rozhkova et al., 1984), and in the cockroach, behavioral compensation has been demonstrated in response to experimentally altered cercal position (Comer and Camhi, 1984). Although not identified in the periphery, there are strong grounds for believing that the PAD unit is a receptor situated at the base of the cercus that monitors the displacement of the cercus from its resting position. The PAD unit responded with discrete bursts of spikes to stepped displacements of the cercus in either direction (Fig. 12). The spikes occurred predominantly on the rising and falling phases of the transducer output, which suggested a response to velocity much like the cuticular hair plate receptor of the lobster antenna (Vedel, 1986). The PAD unit also produced maintained discharges of spikes, and these were evoked particularly by mechanical stimulation of filiform afferents (Figs. 4, 5), sometimes singly. These discharges are similar to those of the chordotonal and joint angle receptors of the locust leg (Usherwood et al., 1968; Kuster and French, 1983; Zill and Forman, 1983), and may have been triggered by reflex contractions of the abdominal musculature, producing movements of the cercus with a longer time course than the movements that produced the phasic response (Fig. 12). Since passive movements of the cercus rarely evoked the maintained type of discharge in the PAD unit, it appears that the PAD unit may be sensitive to more types of motion than could be adequately tested in the experiments described above.

Physiological evidence, such as uniform EPSP size and shape in a postsynaptic cell (Fig. 10A), little summation of activity in a postsynaptic cell (Fig. 10B), and uniform spike height in extraccllular rccordings from the cercal nerve in any one preparation suggest that only one PAD unit exists on each cercus. However, it is not certain from the morphology of central projections and extracellular physiology alone that, although only one PAD unit was being recorded in any given preparation, it was the same unit in every preparation. Indeed, in the cockroach, a chordotonal organ has been reported at the base of each cercus, and the receptors innervating this organ are sensitive to displacements of the cercus in an almost identical fashion to that described above for the locust (Körner et al., 1984). The central projections of these movement-sensitive afferents in the cockroach (Füller et al., 1981) also match those reported here for the locust (Fig. 8), and, although Körner et al. (1984) did not perform experiments to determine a physiological effect on the filiform afferents, Bernard (1987) has reported that afferents, probably from the same chordotonal organ, inhibit synaptic activity in GINs during imposed cercal movements.

\section{Implications for behavior}

Stimulation of cercal afferents and GINs has been demonstrated during cricket stridulation (Kämper and Dambach, 1985) and cockroach walking (Orida and Josephson, 1978; Plummer and Camhi, 1981). The externally generated air currents are much weaker here than during a vigorous behavior such as flight, where phasic air currents reaching the cerci originate from oncoming wind, from the wingbeat (Horsmann et al., 1983), and also from the vibration of the abdomen at the flight frequency (Camhi and Hinkle, 1974). The filiform hairs are therefore subject to a number of influences that constitute noise against which an incoming stimulus must be discriminated. Plummer and Camhi (1981) have proposed that differences in the acceleration properties of wind from various sources allow the cockroach to discriminate peripherally an external signal from walking-induced noise. In addition, there is considerable evidence for a 
corollary discharge from central neurons descending to the terminal ganglion of the cricket and cockroach during walking (Murphey and Palka, 1974; Delcomyn, 1977; Delcomyn and Daley, 1979). This corollary discharge alters the excitability of GINs receiving sensory input from filiform hairs on the cercus (Daley and Delcomyn, 1980a, b). However, in a novel experiment, Orida and Josephson (1978) glued the severed abdomen of a cockroach to the dorsal surface of an intact "carrier" cockroach. By recording from GINs in the dissected abdomen as the "carrier" walked, Orida and Josephson showed that modulation of excitability in the giants still occurred during walking. These results demonstrate a peripheral modulation of GIN excitability, in addition to that mediated by descending units participating in corollary discharge (Delcomyn, 1977; Delcomyn and Daley, 1979). The properties of the PAD unit described for the locust cercal system in the present study would suit it for such a peripherally mediated modulatory function.

If the PAD unit responded to imposed movements of the cercus that were sufficient to stimulate the filiform hairs, such as might occur during flight or jumping, then by presynaptically depolarizing the filiform afferent terminals and blocking spikes, it would ensure that a weaker signal crossed the synapse to postsynaptic cells. The PAD unit would also change the membrane conductance in GINs postsynaptic to filiform afferents. This conductance change could act in an inhibitory manner on the postsynaptic membrane so as to allow fewer spikes to be generated by a given stimulus. In this way, an entire pathway would become desensitized to movement-generated filiform afferent input, but would remain sensitive to external wind stimuli with different acceleration characteristics (Plummer and Camhi, 1981). The cockroach does, in fact, ignore the afferent input from filiform hairs caused by its own movement, and only responds to applied wind to the cerci (Plummer and Camhi, 1981).

\section{References}

Altman, J. (1983) Sensory inputs and the generation of the locust flight motor pattern: From the past to the future. In Biona Report, 2, W. Nachtigall, ed., pp. 127-136, Gustav Fischer, Stuttgart.

Baylor, D. A., and J. G. Nicholls (1969) Chemical and electrical synaptic connexions between cutaneous mechanoreceptor neurones in the central nervous system of the leech. J. Physiol. (Lond.) 203: 591609.

Bell, C. C. (1981) An efference copy which is modified by reafferent input. Science 214: 450-453.

Bernard, J. (1987) Effectiveness of the cercal chordotonal inhibitory organ in the cockroach. Synaptic activity during imposcd ccrcal movements. Comp. Biochem. Physiol. 87 A: 53-56.

Blagburn, J. M., and D. B. Sattelle (1987) Presynaptic depolarization mediates presynaptic inhibition at a synapse between an identified mechanosensory neurone and giant interneurone 3 in the first instar cockroach, Periplaneta americana. J. Exp. Biol. 127: 135-157.

Boyan, G. S. (1986) Modulation of auditory responsiveness in the locust. J. Comp. Physiol. 158: 813-825.

Boyan, G. S., S. Ashman, and E. E. Ball (1986) Initiation and modulation of flight by a single giant interneuron in the cercal system of the locust. Naturwissenschaften 73: 272-274.

Bryan, J. S., and F. B. Krasne (1977) Protection from habituation of the crayfish lateral giant fibre escape response. J. Physiol. (Lond.) 271: 351-368.

Byrne, J. H. (1980) Identification of neurons contributing to presynaptic inhibition in Aplysia californica. Brain Res. 199: 235-239.

Callec, J. J. (1985) Synaptic transmission in the central nervous system. In Comprehensive Insect Physiology, Biochemistry and Pharmacology. Nervous System: Structure and Motor Function vol. 5, chapt. 5, G. A. Kerkut and L. I. Gilbert, eds., pp. 139-179, Pergamon, Oxford.
Camhi, J. M. (1980) The escape system of the cockroach. Sci. Am. 243: 144-157.

Camhi, J. M., and M. Hinkle (1974) Response modification by the central flight oscillator of locusts. J. Exp. Biol. 60: 477-492.

Comer, C., and J. M. Camhi (1984) Behavioral compensation for altered cercal position in the cockroach. J. Comp. Physiol. 155: 3138.

Cook, P. M. (1951) Observations on giant fibres of the nervous system of Locusta migratoria. Q. J. Micro. Sci. 92: 297-305.

Coro, F., and M. Pérez (1983) Peripheral interaction in the tympanic organ of a moth. Naturwissenschaften 70:99-100.

Daley, D. L., and F. Delcomyn (1980a) Modulation of the excitability of cockroach giant interneurons during walking. I. Simultaneous excitation and inhibition. J. Comp. Physiol. 138: 231-239.

Daley, D. L., and F. Delcomyn (1980b) Modulation of the excitability of cockroach giant interneurons during walking. II. Central and peripheral components. J. Comp. Physiol. 138: 241-251.

Dambach, M., H.-G. Rausche, and G. Wendler (1983) Proprioceptive feedback influences the calling song of the field cricket. Naturwissenschaften $70: 417-418$.

Delcomyn, F. (1977) Corollary discharge to cockroach giant interneurones. Nature 269: 160-162.

Delcomyn, F., and D. L. Daley (1979) Central excitation of cockroach giant interneurons during walking. J. Comp. Physiol. 130: 39-48.

Dorsett, D. A. (1980) Design and function of giant fibre systems. Trends Neurosci. 3: 205-208.

Eccles, J. C., R. F. Schmidt, and W. D. Willis (1962) Presynaptic inhibition of the spinal monosynaptic reflex pathway. J. Physiol. (Lond.) 161: 282-297.

Eckert, R. O. (1961) Reflex relationships of the abdominal stretch receptors of the crayfish. I. Feedback inhibition of the receptors. J. Cell. Comp. Physiol. 57: 149-162.

Fahrenbach, W. H. (1985) Anatomical circuitry of lateral inhibition in the eye of the horseshoe crab, Limulus polyphemus. Proc. R. Soc. Lond. [Biol.] 225: 219-249.

Fraser, P. (1977) Cercal ablation modifies tethered flight behaviour of cockroach. Nature 268: 523-524.

Füller, H., A. Ernst, and G. Klare (1981) Iontophoretsche und elektronmikroskopische Untersuchungen der Cercalnerven von Periplaneta americana (L.). Zool. Jb. Anat. 105: 371-405.

Glantz, R. M., L. Wang-Bennett, and B. Waldrop (1985) Presynaptic inhibition in the crayfish brain. I. Inhibition of a central synapse and synaptic events in presynaptic terminals. J. Comp. Physiol. 156:477487.

Horsmann, U., H.-G. Heinzl, and G. Wendler (1983) The phasic influence of self-generated air current modulations on the locust flight motor. J. Comp. Physiol. 150: 427-438.

Hoyle, G. (1958) The leap of the grasshopper. Sci. Am. 198: 30-35.

Huber, F. (1965) Brain controlled behaviour in orthopterans. In The Physiology of the Insect Central Nervous System, J. E. Treherne and J. W. L. Beament, eds., pp. 233-246, Academic, London.

Johnstone, J. R., and R. F. Mark (1971) The efference copy neurone. J. Exp. Biol. 54: 403-414.

Kämper, G. (1984) Abdominal ascending interneurons in crickets: Responses to sound at the $30-\mathrm{Hz}$ calling-song frequency. J. Comp. Physiol. 155: 507-520.

Kämper, G., and M. Dambach (1985) Low-frequency airborne vibrations generated by crickets during singing and aggression. J. Insect Physiol. 31: 925-929.

Kennedy, D., R. L. Calabrese, and J. J. Wine (1974) Presynaptic inhibition: Primary afferent depolarization in crayfish neurons. Science 186: 451-454.

Kirk, M. D. (1985) Presynaptic inhibition in the crayfish CNS: Pathways and synaptic mechanisms. J. Neurophysiol. 54: 1305-1325.

Kirk, M. D., and J. J. Wine (1984) Identified interneurons produce both primary afferent depolarization and presynaptic inhibition. Science 225: 854-856.

Körner, U., E. Körner, and R. Ehrlich (1984) Elektrophysiologische Untersuchung eines pericercalen Chordotonalorgans der Schabe Periplaneta americana (L.). Zool. Jb. Physiol. 88: 147-164.

Krasne, F. B., and J. S. Bryan (1973) Habituation: Regulation through presynaptic inhibition. Science 182: 590-592.

Kretz, R., E. Shapiro, and E. R. Kandel (1986a) Presynaptic inhibition produced by an identified presynaptic inhibitory neuron. I. Physiological mechanisms. J. Neurophysiol. 55: 113-130. 
Kretz, R., E. Shapiro, C. H. Bailey, M. Chen, and E. R. Kandel (1986b) Presynaptic inhibition produced by an identified presynaptic inhibitory neuron. II. Presynaptic conductance changes caused by histamine. I. Neurophysiol. 55: 131-146.

Kuster, J. E., and A. S. French (1983) Sensory transduction in a locust multipolar joint receptor: The dynamic behaviour under a variety of stimulus conditions. J. Comp. Physiol. 150: 207-215.

Levine, R. B., and R. K. Murphey (1980) Pre- and postsynaptic inhibition of identified giant interneurons in the cricket (Acheta domesticus). J. Comp. Physiol. 135: 269-282.

Levy, R. A. (1977) The role of GABA in primary afferent depolarization. Prog. Neurobiol. 9: 211-260.

Montgomery, J. C. (1984) Noise cancellation in the electrosensory system of the thornback ray; common mode rejection of input produced by the animal's own ventilatory movement. J. Comp. Physiol. 155: 103-111.

Murphey, R. K., and J. Palka (1974) Efferent control of cricket giant fibres. Nature 248: 249-251.

Nicoll, R. A., and B. E. Alger (1979) Presynaptic inhibition: Transmitter and ionic mechanisms. Int. Rev. Neurobiol. 21: 217-256.

Orida, N., and R. K. Josephson (1978) Peripheral control of responsiveness to auditory stimuli in giant fibres of crickets and cockroaches. J. Exp. Biol. 72: 153-164.

O'Shea, M., and C. H. F. Rowell (1975) Protection from habituation by lateral inhibition. Nature 254: 53-55.

Palka, J. (1967) An inhibitory process influencing visual responses in a fibre of the ventral nerve cord of locusts. J. Insect Physiol. 13: 235248.

Palka, J. (1969) Discrimination between movements of eye and object by visual interneurones of crickets. J. Exp. Biol. 50: 723-732.

Pearson, K. G., and C. S. Goodman (1981) Presynaptic inhibition of transmission from identified interneurons in locust central nervous system. J. Neurophysiol. 45: 501-515.

Pearson, K. G., G. S. Boyan, M. Bastiani, and C. S. Goodman (1985) Heterogeneous properties of segmentally homologous interneurons in the ventral nerve cord of locusts. J. Comp. Neurol. 233: 133-145.

Plummer, M. R., and J. M. Camhi (1981) Discrimination of sensory signals from noise in the escape system of the cockroach: The role of wind acceleration. J. Comp. Physiol. 142: 347-357.

Rozhkova, G. I. (1980) Comparison of the constancy mechanisms in the cercal systems of crickets (Acheta domesticus and Gryllus bimaculatus). J. Comp. Physiol. 137: 287-296.

Rozhkova, G. I., H. I. Rodionova, and A. V. Popov (1984) Two types of information processing in cercal systems of insects: Directional sensitivity of giant interneurons. J. Comp. Physiol. 154: 805-815.
Russell, I. J., and B. L. Roberts (1974) Active reduction of lateral-line sensitivity in swimming dogfish. J. Comp. Physiol. 94: 7-15.

Ryall, R. W. (1978) Presynaptic inhibition. Trends Neurosci. 1: 164166.

Sandeman, D. C. (1968) A sensitive position measuring device for biological systems. Comp. Biochem. Physiol. 24: 635-638.

Seabrook, W. D. (1970) The structure of the terminal ganglionic mass of the locust, Schistocerca gregaria (Forskal). J. Comp. Neurol. 138: $63-86$.

Seabrook, W. D. (1971) An electrophysiological study of the giant fiber system of the locust Schistocerca gregaria. Can. J. Zool. 49:555560.

Sihler, H. (1924) Die Sinnesorgane an den Cerci der Insekten. Zool. Jb. Anat. 45: 519-580.

Solodkin, M., I. Jiménez, and P. Rudomin (1984) Identification of common interneurons mediating pre- and postsynaptic inhibition in the cat spinal cord. Science 224: 1453-1456.

Thomas, J. G. (1965) The abdomen of the female desert locust (Schistocerca gregaria Forskal) with special reference to the sense organs. Anti-Locust Bull. 42: 1-20.

Usherwood, P.N. R., H. I. Runion, and J. I. Campbell (1968) Structure and physiology of a chordotonal organ in the locust leg. J. Exp. Biol. 48: 305-323.

Uvarov, B. (1966) Grasshoppers and locusts, vol. I. Anatomy, Physiology, Development, Phase Polymorphism, Introduction to Taxono$m y$, Cambridge U. P., Cambridge.

Vedel, J.-P. (1986) Morphology and physiology of a hair plate sensory organ located on the antenna of the rock lobster Palinurus vulgaris. J. Neurobiol, 17: 65-76.

von Holst, E., and H. Mittelstaedt (1950) Das Reafferenzprinzip. Naturwissenschaften $37:$ 464-476.

Wang-Bennett, L. T., and R. M. Glantz (1985) Presynaptic inhibition in the crayfish brain. II. Morphology and ultrastructure of the terminal arborization. J. Comp. Physiol. 156: 605-617.

Wang-Bennett, L. T., and R. M. Glantz (1986) Integration and spike initiation in neuronal terminals. J. Neurosci. 6: 1726-1732.

Watson, A. H. D., and H.-J. Pflüger (1984) The ultrastructure of prosternal sensory hair afferents within the locust central nervous system. Neuroscience 11:269-279.

Zill, S. N., and R. R. Forman (1983) Proprioceptive reflexes change when an insect assumes an active, learned posture. J. Exp. Biol. 107: 385-390. 